\title{
Empleo simultáneo de la fotografía digital y datos de campo para el seguimiento del estado fenológico y el consumo hídrico en lechuga (Lactuca Sativa L CV Neruda) con distintos tratamientos de suelo
}

\author{
A. Fernández López¹, M. Soler-Méndez², J.M. Molina-Martínez², G. García Mateos³ , A. Ruiz- \\ Canales $^{1}$ \\ 1 Departamento de Ingeniería, Universidad Miguel Hernández de Elche, Escuela Politécnica Superior de \\ Orihuela (EPSO-UMH). Crtra. de Beniel, km 3,2. 03312 Orihuela (Alicante), Spain. \\ 2 Grupo de I+D+i de Ingeniería Agromótica y del Mar. UPCT. C/ del Ángel s/n. Ed. ELDI E1.06.30202 \\ Cartagena (Murcia), Spain \\ 3 Departamento de Informática y Sistemas. Área de Lenguajes y Sistemas. Facultad de Informática. \\ Universidad de Murcia. Campus de Espinardo, 30100 Murcia, Spain
}

\begin{abstract}
Resumen: Se desarrollaron los ensayos en una parcela experimental de la Escuela Politécnica Superior de Orihuela (EPSO) de la Universidad Miguel Hernández de Elche (UMH). Las diferencias entre tratamientos dependían de la adición de diferentes tipos de enmiendas y abonado en el suelo. Se escogió como cultivo de ensayo la lechuga (Lactuca Sativa L) pues tiene un ciclo corto y se pueden obtener resultados de una manera relativamente rápida y sencilla. Se realizó a lo largo de dos campañas un seguimiento de fotografías cenitales con cuatro repeticiones por tratamiento. Mediante la evolución de imágenes cenitales de las dos cosechas de lechugas se analizaron las imágenes 2D mediante software CAPS para calcular los diámetros de la planta y el porcentaje de cobertura vegetal (PGC) a lo largo del tiempo. Durante las tres campañas del experimento, además del cálculo de los diámetros, se midieron simultáneamente, la altura de la planta, la profundidad de la raíz y el peso de la cosecha. En la segunda campaña y desde la nascencia hasta mitad de campaña, el tratamiento T1 tiene valores de diámetro de planta superiores al resto. El T2 en todo momento está por debajo de los demás tratamientos. Se infiere la influencia de la adición de harina de pórfido en los primeros estadios de crecimiento. Esto se corrobora con los valores de altura de planta y no así con los valores de profundidad de raíz. Igualmente, en la segunda campaña y con las medidas de ejemplares a lo largo del experimento se diferencia el tratamiento T1 respecto al resto.
\end{abstract}

Palabras clave: PGC, consumo de agua en riego, CAPS, riego localizado, horticultura

\section{Introducción}

El conocimiento de las necesidades hídricas de los cultivos posibilita conseguir una gestión adecuada del agua de riego con la máxima eficiencia [1]. Mediante la estimación del balance hídrico a escala de cultivo se establece la demanda de agua a lo largo del ciclo de vida del cultivo [2]. Este balance se obtiene estimando las entradas y salidas de agua que se producen a escala del cultivo y añadiendo por riego las cantidades perdidas.

El empleo de imágenes de cobertura vegetal a la estimación de las necesidades hídricas de los cultivos está basado en la determinación de la fracción de cobertura del dosel vegetal [3]. La fracción de cobertura vegetal (PGC) permite estimar las necesidades hídricas de los cultivos al 


\section{CONGRESO IBÉRICO DE AGROINGENIERÍA \\ X CONGRESSO IBÉRICO DE AGROENGENHARIA \\ 3 - 6 septiembre 2019, Huesca - España}

estar directamente relacionado con la evapotranspiración (ETc), que se relaciona con el coeficiente de cultivo (Kc) y la evapotranspiración de referencia (ETo) [4]. En lechuga, se establecieron métodos basados en el procesamiento digital de imágenes para estimar el coeficiente de cultivo a partir de fotografías digitales de la cobertura vegetal [5].

El objetivo del presente trabajo es el seguimiento del estado fenológico y el consumo hídrico de un cultivo de lechuga (Lactuca sativa L. cv. Neruda) con tres tipos de combinación de fertilizante y de enmienda del suelo. Para calcular la cubierta vegetal se empleó fotografía digital y técnicas de procesamiento digital de imágenes. Se simultanearon los datos de la fotografía digital con medidas de planta (altura de planta, profundidad radical), consumo hídrico (riego) y lluvia y peso de la cosecha.

\section{Materiales y métodos}

Se desarrollaron los ensayos durante dos campañas (del 03/10/2016 al 12/12/2016 y del 23/02/2017 al 04/05/2017) en una parcela experimental de $300 \mathrm{~m}^{2}$ de la Escuela Politécnica Superior de Orihuela (EPSO) de la Universidad Miguel Hernández de Elche (UMH). Se estudiaron cuatro tratamientos diferenciales respecto a las propiedades del suelo de cultivo. Los datos presentados en esta comunicación se incluyen dentro de un estudio más amplio que fue el estudio del efecto en las propiedades texturales y edáficas del suelo, así como sus efectos en la producción hortícola, de la adición de harina de roca de pórfido, material desarrollado por la empresa Fulsan, S.A., a un suelo agrícola. Se escogió como cultivo de estudio la lechuga (Lactuca Sativa L Neruda) pues tiene un ciclo corto y se obtienen resultados de una manera relativamente rápida y sencilla.

En el primer tratamiento (T1) se le añadió al suelo al inicio de la campaña una cantidad convenida de harina de pórfido y se aplicó junto con un abonado de fondo inorgánico. Durante las dos campañas de riego de los dos ciclos de cultivo se le aplicó al correspondiente sector (T1) de la plantación de lechuga, además de las correspondientes dosis de riego establecidas, un abonado inorgánico (fertirriego).

Para el segundo tratamiento (T2) se mezclaron harina de pórfido Fulsan y compost (Bocashi) con el suelo agrícola de la parcela experimental y esta mezcla constituyó un abonado de fondo orgánico. Durante las dos campañas de riego se le aplicó al correspondiente sector (T2) de la plantación de lechuga, además de las correspondientes dosis de riego establecidas, un fertirriego orgánico. El tratamiento T2 no recibió fertirriego inorgánico.

Para el tercer tratamiento (T3) no se añadió ninguna sustancia inicialmente al suelo agrícola. Durante las dos campañas de riego de los dos ciclos de cultivo se le aplicó al correspondiente sector (T3) de la plantación de lechuga, además de las correspondientes dosis de riego establecidas, un abonado inorgánico (fertirriego), como en el tratamiento T1.

Adicionalmente se consideró incluir un cuarto tratamiento control (T4) para compararlo con los tratamientos anteriores. A este último tratamiento no se añadió abonado de fondo ni se le añadió fertirriego.

Se realizaron a lo largo de las dos campañas un seguimiento de fotografías cenitales con cuatro repeticiones por tratamiento. Mediante la evolución de imágenes cenitales de las dos cosechas de lechugas se analizaron las imágenes 2D mediante software CAPS ${ }^{\circledR}$ para calcular los diámetros de la planta y el porcentaje de cobertura vegetal (PGC) a lo largo del tiempo (Fig. 1). El software citado permite diferenciar en una fotografía, la zona de tierra de la zona de planta. De este modo se delimita cada una de las zonas a la vez que se determinan las superficies ocupadas por cada zona. 

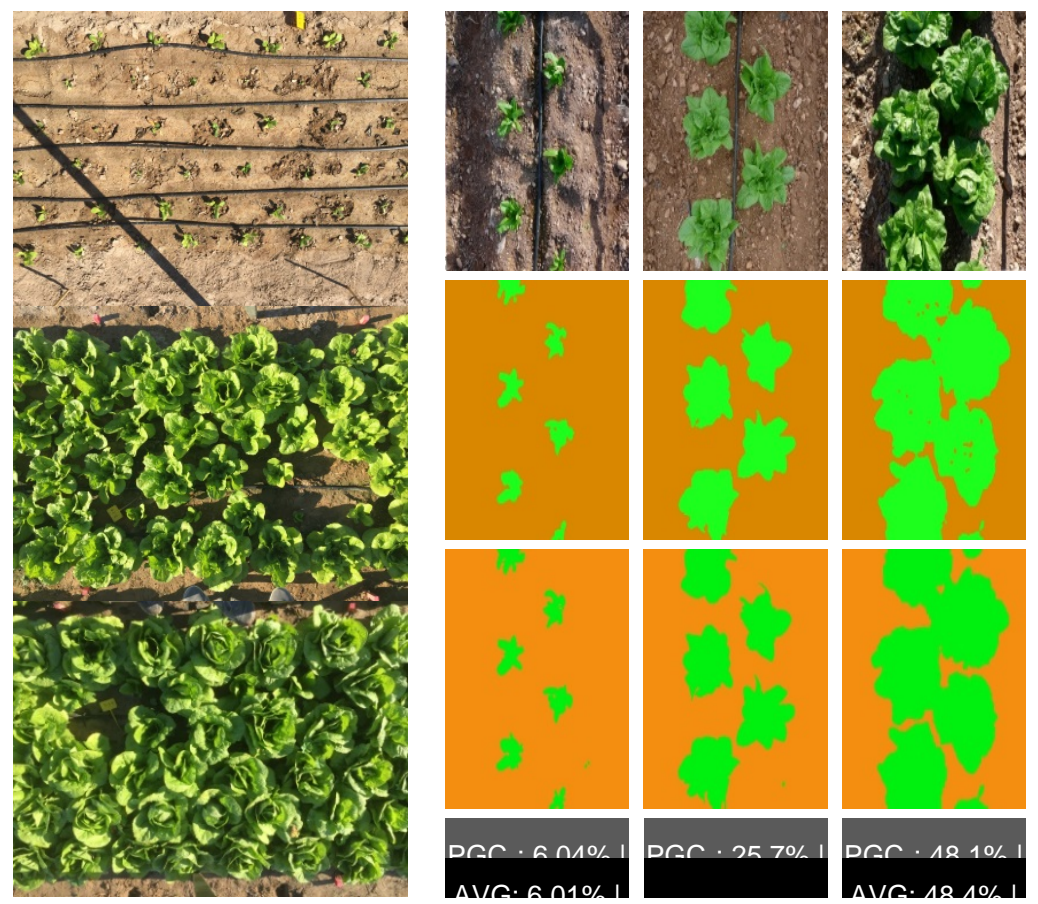

Figura 1. Metodología empleada para la determinación del porcentaje de cubierta sombreada mediante fotografía digital. A la izquierda las imágenes cenitales del marco de plantación en diferentes estados de crecimiento. A la derecha, mediante el software CAPS, se determina la zona correspondiente con la cubierta vegetal del cultivo de lechuga frente a la zona de tierra y elementos ajenos a la planta. Esta información se contrasta con los registros de los consumos de agua.

Durante las dos campañas del experimento, además del cálculo de los diámetros, se midieron en campo y en determinadas fechas, la altura de la planta, la profundidad de la raíz y el agua aplicada en riego y la lluvia. Simultáneamente se midió el peso de la cosecha en ambas campañas. Se muestran cada uno de los parámetros medidos en los apartados siguientes.

\section{Resultados y discusión}

En la segunda campaña y desde la nascencia hasta mitad de campaña, el tratamiento T1 tiene valores de diámetro de planta superiores al resto. El T2 en todo momento está por debajo de los demás tratamientos. Se puede inferir una cierta influencia de la adición de harina de pórfido en los primeros estadios de crecimiento. Esto se corrobora con los valores de altura de planta y no así con los valores de profundidad de raíz. Igualmente, en la segunda campaña y con las medidas de ejemplares a lo largo del experimento se diferencia el tratamiento T1 respecto al resto.

El peso medio de la cosecha (por planta) para la primera campaña fue el siguiente:

Tratamiento T1: $1,195 \mathrm{~kg} \pm 0,303$

Tratamiento T2: $1,014 \mathrm{~kg} \pm 0,150$

Tratamiento T3: $1,249 \mathrm{~kg} \pm 0,259$

Tratamiento T4: $1,077 \mathrm{~kg} \pm 0,172$

El peso medio de la cosecha para la segunda campaña fue el siguiente:

Tratamiento T1: $0,758 \mathrm{~kg} \pm 0,192$

Tratamiento T2: $0,788 \mathrm{~kg} \pm 0,196$

Tratamiento T3: $1,076 \mathrm{~kg} \pm 0,222$

Tratamiento T4: 0,896 kg $\pm 0,199$ 


\section{CONGRESO IBÉRICO DE AGROINGENIERÍA \\ X CONGRESSO IBÉRICO DE AGROENGENHARIA \\ 3 - 6 septiembre 2019, Huesca - España}

Según lo expuesto, la producción en la segunda campaña fue de menor cuantía, alrededor de un $3 \%$ menor.

Los valores de peso de cosecha (peso por planta) mostraron que en la primera cosecha se obtuvo el mayor peso en el tratamiento T3, seguido del tratamiento T1, T2 y T4.

Para la segunda cosecha, el mayor peso se dio en el tratamiento T3, seguido de T4, T2 y T1. Los valores de la segunda cosecha fueron un $3 \%$ menores.

Hay una cierta estabilización de la homogeneización del estado vegetativo respecto al riego en la segunda campaña, aunque los valores de cosecha han mostrado peores resultados. Puede que la influencia en los parámetros de cosecha se muestre en las siguientes cosechas ya que el suelo ha almacenado una serie de elementos y compuestos.

\section{Primera campaña}

Se muestra en la figura 2 la evolución del diámetro medio de la planta para la primera campaña. A lo largo de la primera campaña (del 03/10/2016 al 12/12/2016) los diámetros medios del T1 y T2 son inferiores al T3 y T4.

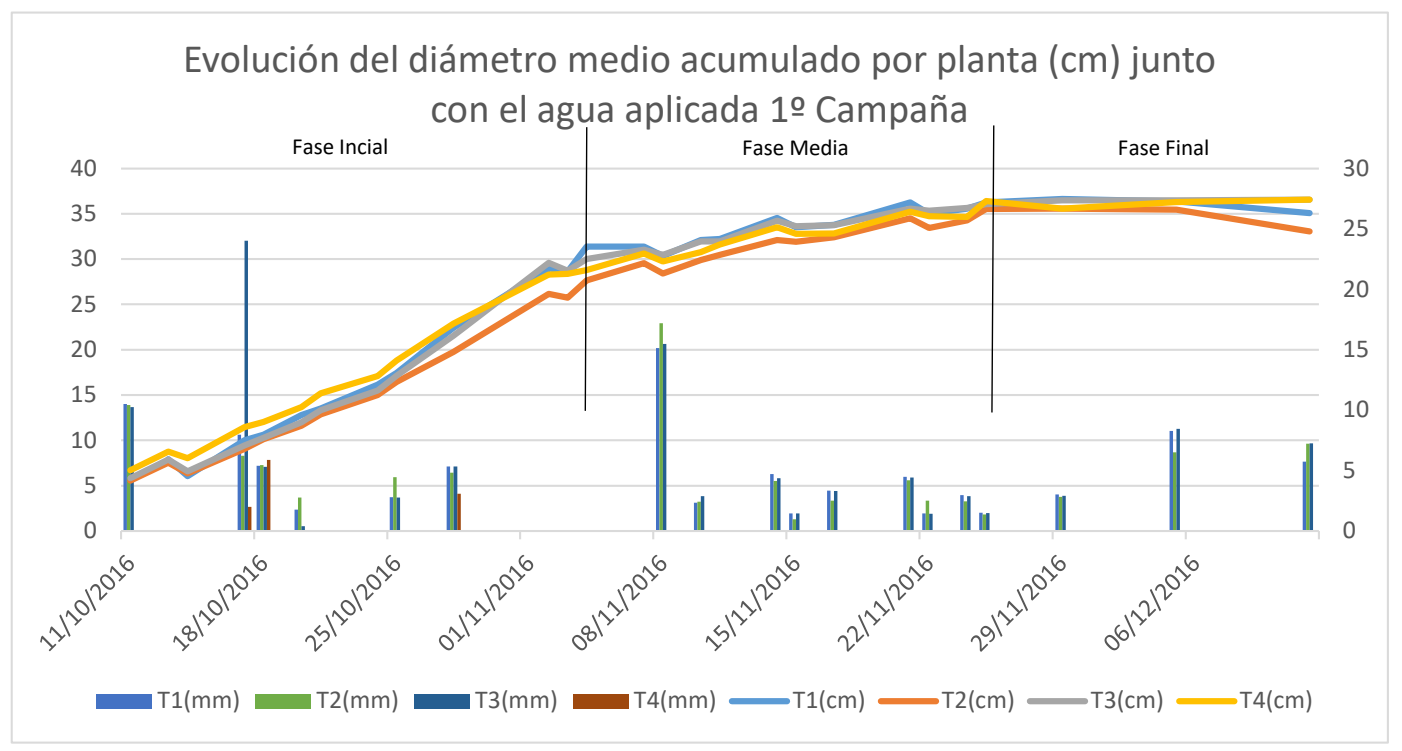

Figura 2. Comparativa de las medidas de diámetro medio mediante análisis de fotografía cenital para los cuatro tratamientos T1, T2, T3 y T4 durante la primera campaña (del 03/10/2016 al 12/12/2016). Se incluyen las cantidades de agua de riego aplicadas y lluvia caída.

\section{Segunda campaña}

En la figura 3 comienzan los tratamientos T1 y T2 a evolucionar con valores superiores a T3 y T4 pero a medida que avanza el experimento, el diámetro del T3 es superior al resto. 
X CONGRESO IBÉRICO DE AGROINGENIERÍA

X CONGRESSO IBÉRICO DE AGROENGENHARIA

3 - 6 septiembre 2019, Huesca - España

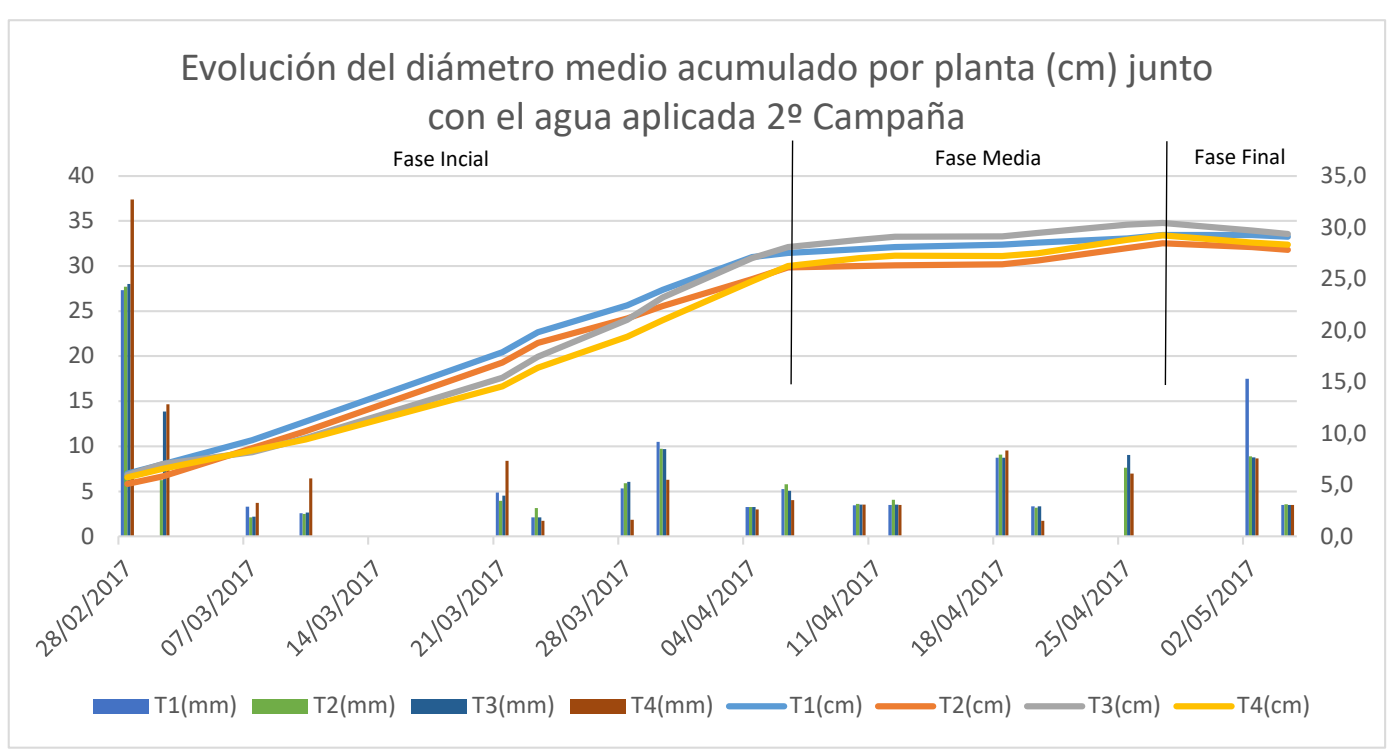

Figura 3. Comparativa de las medidas de diámetro medio mediante análisis de fotografía cenital para los cuatro tratamientos T1, T2, T3 y T4 durante la segunda campaña (del 23/02/2017 al 04/05/2017). Se incluyen las cantidades de agua de riego aplicada y lluvia caída.

Se muestra una cierta diferenciación en la segunda campaña a favor de los tratamientos T1 y T2 que al final del experimento no se da en el T2.

Los datos de la evolución de la altura de la planta se tomaron a lo largo del período experimental en determinadas fechas. Las figuras 4 y 5 reflejan la evolución de este parámetro medido directamente en campo.

\section{Primera campaña}

En la figura 4 se muestra que el tratamiento T1 tiene una altura mayor que el resto, pero el T2 se muestra en todo el período experimental por debajo del resto de tratamientos. T3 y T4 presentan valores intermedios.

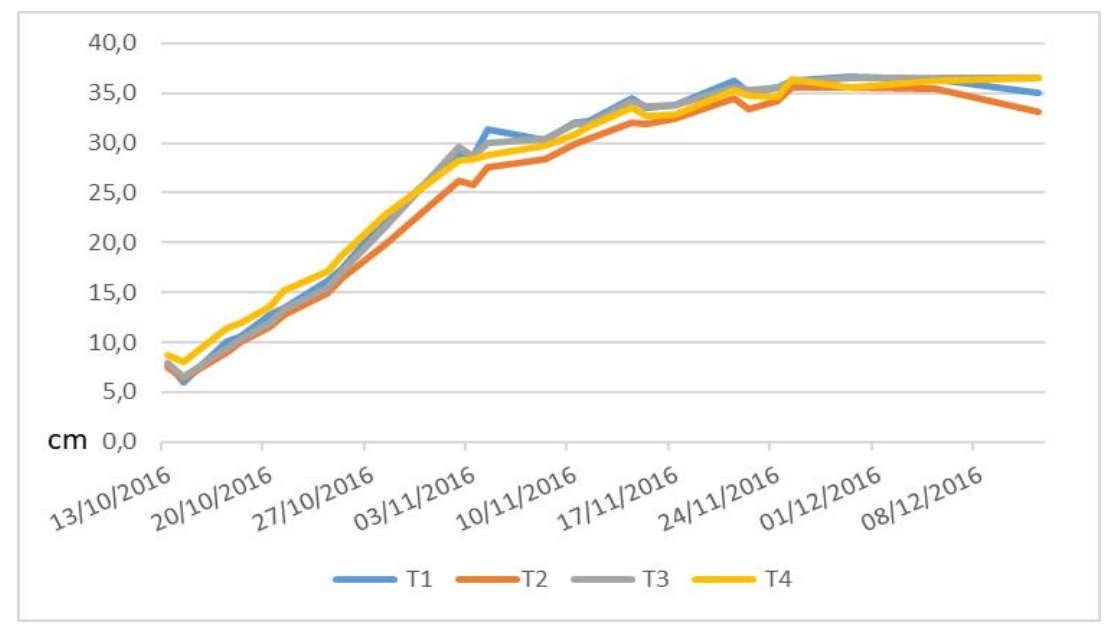

Figura 4. Evolución de las medidas de la altura media de la planta en cm mediante medición directa en campo para los cuatro tratamientos T1, T2, T3 y T4 durante la primera campaña (del 03/10/2016 al 12/12/2016). 


\section{CONGRESO IBÉRICO DE AGROINGENIERÍA \\ X CONGRESSO IBÉRICO DE AGROENGENHARIA \\ 3 - 6 septiembre 2019, Huesca - España}

\section{Segunda campaña}

La evolución de las medidas de altura media de la planta durante la segunda campaña queda reflejada en la figura 5. En esta campaña, la tendencia de T1 y T2 ha sido superior a T3 y T4 desde el inicio hasta mitad de campaña. A partir de ese momento se invirtió la tendencia y los valores más elevados de altura media de planta correspondían a T3 y T4. Globalmente los valores han evolucionado de una manera similar en las dos campañas, aunque se hayan alcanzado algunos valores superiores puntuales en la segunda campaña.

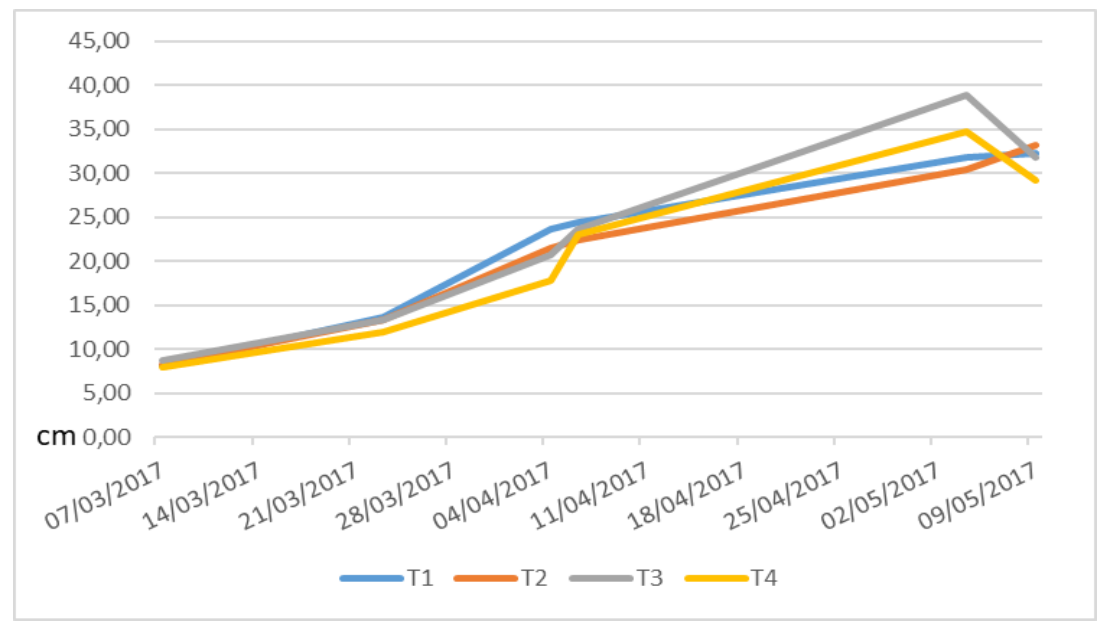

Figura 5. Evolución de las medidas de la altura media de la planta en cm mediante medición directa en campo para los cuatro tratamientos T1, T2, T3 y T4 durante la segunda campaña (del 23/02/2017 al 04/05/2017).

Los datos de la evolución de la profundidad de la raíz se midieron en campo a lo largo del período experimental. En las figuras 6 y 7 se muestran los resultados correspondientes.

\section{Primera campaña}

En la figura 6 se muestra que el tratamiento T1 y T2 tuvieron una tendencia a alcanzar mayores valores en la parte final del experimento, mientras que al principio ocuparon posiciones de menor valor.

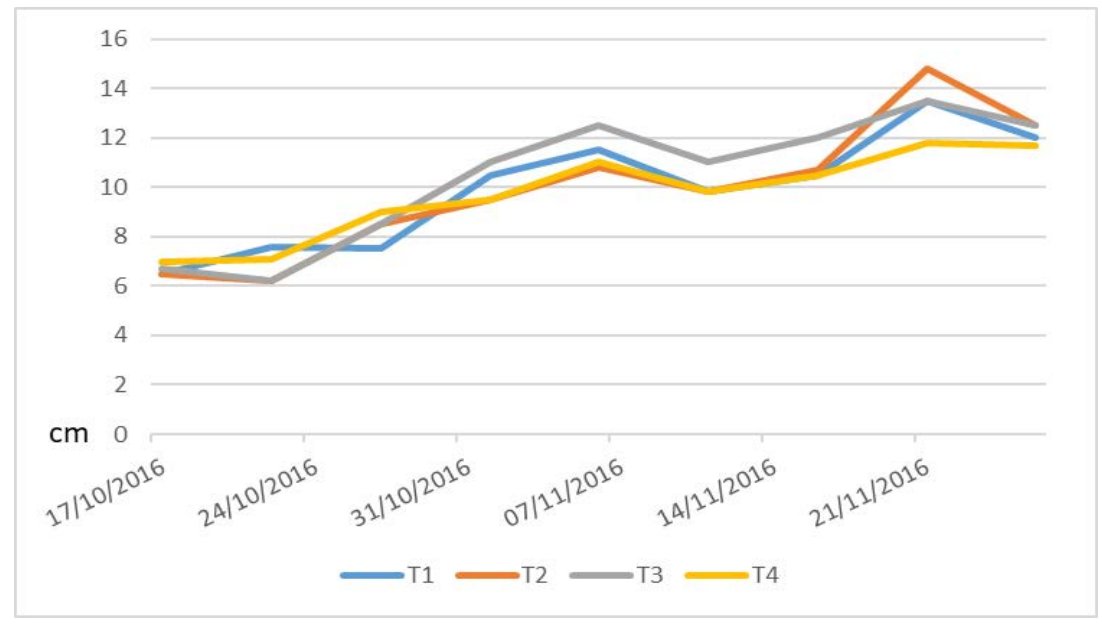

Figura 6. Evolución de las medidas de la profundidad media de raíz en cm mediante medición directa en campo para los cuatro tratamientos T1, T2, T3 y T4 durante la primera campaña (del 03/10/2016 al 12/12/2016). 


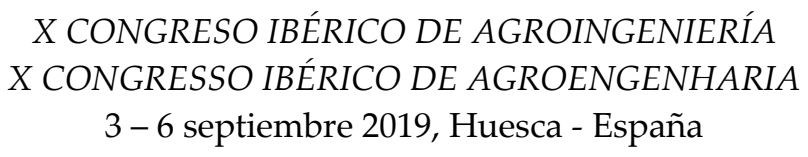

\section{Segunda campaña}

En la figura 7 se muestra que el tratamiento T1 y T2 tuvieron una tendencia a alcanzar mayores valores en la parte inicial del experimento, mientras que al final ocuparon las posiciones de menor valor.

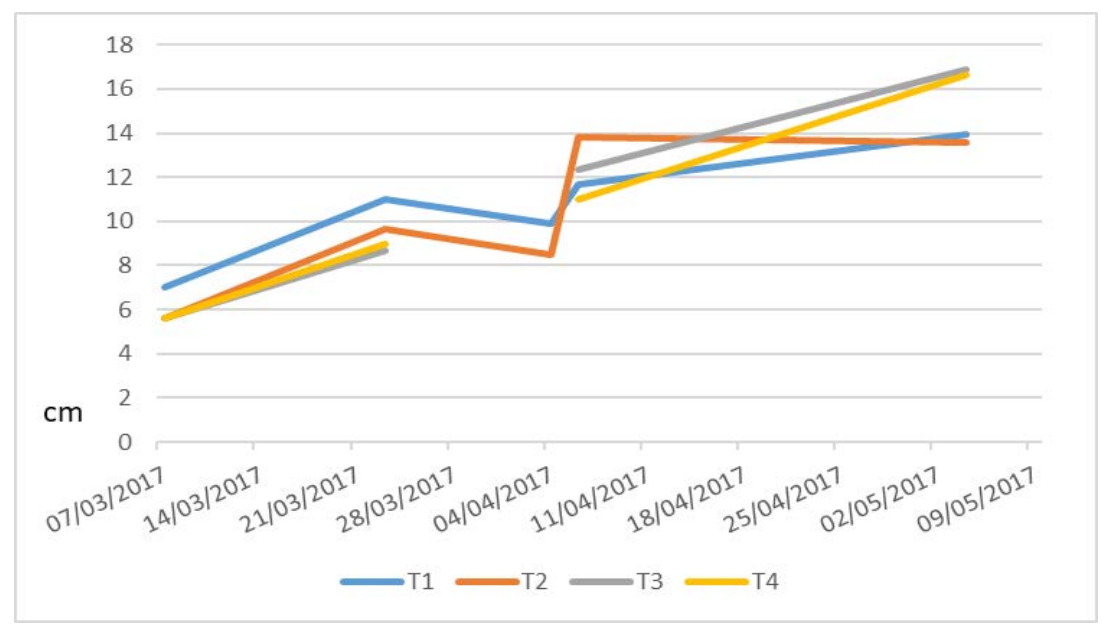

Figura 7. Evolución de las medidas de la profundidad media de raíz en cm mediante medición directa en campo para los cuatro tratamientos T1, T2, T3 y T4 durante la segunda campaña (del 23/02/2017 al 04/05/2017).

\section{Conclusiones}

En la segunda campaña y desde la nascencia hasta mitad de campaña, el tratamiento T1 tiene valores de diámetro de planta superiores al resto. El T2 en todo momento está por debajo de los demás tratamientos. Se puede inferir una cierta influencia de la adición de harina de pórfido en los primeros estadios de crecimiento. Esto se corrobora con los valores de altura de planta y no así con los valores de profundidad de raíz. Igualmente, en la segunda campaña y con las medidas de ejemplares a lo largo del experimento se diferencia el tratamiento T1 respecto al resto.

Los valores de peso de cosecha (peso por planta) mostraron que en la primera cosecha se obtuvo el mayor peso en el tratamiento T3, seguido del tratamiento T1, T2 y T4.

Para la segunda cosecha, el mayor peso se dio en el tratamiento T3, seguido de T4, T2 y T1. Los valores de la segunda cosecha fueron un $3 \%$ menores.

Hay una cierta estabilización de la homogeneización del estado vegetativo respecto al riego en la segunda campaña, aunque los valores de cosecha han mostrado peores resultados. Puede que la influencia en los parámetros de cosecha se muestre en las siguientes cosechas ya que el suelo ha almacenado una serie de elementos y compuestos.

\section{Agradecimientos}

Los autores de la comunicación expresan su agradecimiento a la empresa FULSAN, S.A. por su apoyo económico. Igualmente se muestran agradecidos a la empresa TELENATURA EBT, S.L. y Viveros Simón Cases, S.L. por su apoyo tecnológico y agronómico. 


\section{CONGRESO IBÉRICO DE AGROINGENIERÍA \\ $X$ CONGRESSO IBÉRICO DE AGROENGENHARIA \\ 3 - 6 septiembre 2019, Huesca - España}

\section{Referencias}

1. Novoa, V., Ahumada-Rudolph, R., Rojas, O., Saez, K., de la Barrera, F., Arumi, J.L. Understanding agricultural water footprint variability to improve water management in Chile. Science of the Total Environment, 2019, 670 (188-199).

2. Golzar, F., Heeren, N., Hellweg, S., Roshandel, R. A comparative study on the environmental impact of greenhouses: A probabilistic approach. The Science of the total environment, 2019, 675 (560-569).

3. Ilha, P., Rosso, S., Schiesari, L. Effects of deforestation on headwater stream fish assemblages in the Upper Xingu River Basin, Southeastern Amazonia. Neotropical Ichthyology, 2019, 17, 1, e180099.

4. Gong, X.W., Liu, H., Sun, J.S., Gao, Y., Zhang, H. Comparison of Shuttleworth-Wallace model and dual crop coefficient method for estimating evapotranspiration of tomato cultivated in a solar greenhouse. Agricultural Water Management, 2019, 217 (141-153).

5. Chávez Castillo, E., Paz Pellat, F., Bolaños González, M. A. Estimation of biomass and aerial cover using radiometry and field level digital imaging of grasslands and scrublands. Terra Latinoamericana, 2017, 35 (3), (247-257). 